\title{
Predictors of Mortality in Patients with COVID-19 Infection-associated Acute Kidney Injury
}

\author{
Aysel Tocoglu ${ }^{1}$, Hamad Dheir ${ }^{2}$, Melike Bektas ${ }^{1}$, Seyyid Bilal Acikgoz ${ }^{3}$, Oguz Karabay ${ }^{4}$ and Savas Sipahi ${ }^{1}$ \\ ${ }^{1}$ Department of Internal Medicine, Sakarya University Education and Training Hospital, Sakarya, Turkey \\ ${ }^{2}$ Department of Nephrology, Sakarya University Education and Training Hospital, Sakarya, Turkey \\ ${ }^{3}$ Department of Internal Medicine, 19 Mayss University Medical Faculty, Samsun, Turkey \\ ${ }^{4}$ Department of Infectious Diseases, Sakarya University Education and Training Hospital, Sakarya, Turkey
}

\begin{abstract}
Objective: To determine clinical characteristics, renal replacement therapy (RRT) requirements, and predictors of mortality in critically ill patients with COVID-19 associated AKI.

Study Design: Descriptive study.

Place and Duration of Study: Sakarya University Education and Training Hospital, Sakarya, Turkey, between April 1 and 30 , 2020.

Methodology: The study included 55 patients who were admitted with diagnosis of COVID-19, and whose illnesses showed a critical course that leads to AKI. The variables were studied as per objective.

Results: During the follow-up, 43 out of 55 patients (78.2\%) died and $12(21.8 \%)$ were discharged with recovery. The mortality was higher in patients at stage $3(88.9 \%$ mortality) compared to stage $2(53.8 \%$ mortality) $(p=0.014)$. In the nonsurvivor group, RDW (red cell distribution width) and albumin levels were lower at admission; whereas, the LDH levels and CRP/albumin ratios were higher. On regression analysis, low albumin level (OR: 12.793, p =0.010), high LDH level (OR: 8.454, p = 0.026), and presence of stage 3 AKI (OR: 10.268, $p=0.020$ ) were found as independent risk factors for mortality in COVID-19 patients, who developed AKI.

Conclusion: In critically ill patients with COVID-19 pneumonia, who developed AKI, it was seen that the presence of low albumin, high LDH, and stage $3 \mathrm{AKI}$ at the time of admission could be used as predictors of mortality. Moreover,, it was shown for the first time that in these patients, the high CRP/albumin ratio and low RDW could be associated with mortality.
\end{abstract}

Key Words: Acute kidney injury, Mortality, COVID-19.

How to cite this article: Tocoglu A, Dheir H, Bektas M, Acikgoz SB, Karabay O, Sipahi S. Predictors of Mortality in Patients with COVID-19 Infection-associated Acute Kidney Injury. J Coll Physicians Surg Pak 2021; 31(JCPSPCR):CR60-CR65.

\section{INTRODUCTION}

Coronavirus disease-19 (COVID-19) is a potentially lethal infectious disease caused by severeacuterespiratory syndrome-coronavirus-2 (SARS-CoV-2) and characterised by acute alveolar injury and respiratory failure. Although the lung is the primary target of COVID-19, the disease may involve other systems or organs including kidney, heart, and alimentary, blood, or nervous systems. ${ }^{1}$

Acute kidney injury (AKI) is a significant complication of COVID-19 disease, developed in approximately $5 \%$ of patients admitted to the hospital and $50 \%$ of the patients in the intensive care unit. ${ }^{2}$

Correspondence to: Dr. Aysel Tocoglu, Department of Internal Medicine, Sakarya University Education and Training Hospital, Sakarya, Turkey

E-mail: agurkan@sakarya.edu.tr

Received: November 10, 2020; Revised: January 16, 2021; Accepted: February 19, 2021

DOI: https://doi.org/10.29271/jcpsp.2021.JCPSPCR.CR60
Initial studies showed relatively low rates between 0.5 - 9\%; whereas, subsequent studies revealed that the rates may be as high as $36.6 \% .^{3,4}$ Despite the exact mechanism is unknown, three hypotheses are proposed in order to elucidate how SARS-CoV-2 causes kidney injury. In the first mechanism the SARS-CoV-2, similar to the SARS-CoV virus, enters the cell by binding to the angiotensin converting enzyme (ACE) 2 , which is located on the tubule epithelial cell and podocyte, and which acts as the host cell receptor. The virus may then injure these cells by a direct cytopathic effect and consequently disrupts the acidbase and electrolyte homeostasis. ${ }^{2,5}$ In the second mechanism, the accumulation of immune complexes, formed in response to the viral antigen, may cause renal injury. In the third mechanism, cytokines released in response to sepsis may induce kidney injury by indirect means including hypoxia, shock, and rhabdomyolysis. ${ }^{6,7}$

AKI carries a very high mortality, even in the absence of COVID-19 infection. It is more common in patients with severe disease and carries a negative prognostic value with respect to hospitalisation and survival in ICU. ${ }^{6}$ Predicting the development 
of $A K I$ and the need for renal replacement therapy in patients infected with SARS-CoV-2, it may enable utilisation of protective measures, which can consequently increase the survival rate and shorten the hospitalisation period. In this study, the aim was to determine the clinical characteristics of patients, who developed COVID-19 associated AKI, requirements for renal replacement therapy, and the predictors of mortality.

\section{METHODOLOGY}

The study included critical ill COVID-19 patients who received inpatient treatment at Sakarya University Education and Training Hospital, Sakarya, Turkey, between April 1 and 30, 2020, whose conditions progressed to AKI and necessitated nephrology consultation for renal replacement treatment.

COVID-19 was diagnosed with the real-time polymerase chain reaction ( $R T-P C R$ ) analysis of samples obtained by nasopharyngeal swab (NPSW). The severity of the disease was classified according to the Chinese Clinical Guidance for COVID-19 Pneumonia Diagnosis and Treatment. ${ }^{8} \mathrm{AKI}$ was defined and staged (stage 1, 2, and 3), based on the KDIGO Clinical Practice Guideline for Acute Kidney Injury. ${ }^{9}$ Patients under the age of 18 years, with known chronic renal failure (CRF), congestive heart disease (CHD), history of malignancy, unknown basal serum creatinine levelsor pregnancy, were excluded.

Creatinine levels measured within the previous 3 months were defined as basal, creatinine levels measured at admission to the emergency unit were defined as admission, and the highestcreatinine levels at admission or during hospitalisation were defined as peak creatinine levels. Based on creatinine levels at admission, patients were separated into two groups: normal and high. Other laboratory values analysed in the study were obtained from studies performed at admission.

The data were assessed by using the SPSS version 22.0 (SPSS, Chicago. IL). For data assessment, Kolmogorov-Smirnov or Shapiro-Wilk test was used to determine whether the variables show a normal distribution or not. Central distribution criteria of normally distributed numerical variables were specified as mean \pm standard deviation and the numerical variables, not showing normal distribution, was specified as median $\left(25^{\text {th }}\right.$ percentile- $75^{\text {th }}$ percentile). Categorical data were expressed as percentage (\%). Student-t test or Mann-Whitney U-test was used for the comparison of between-group differences in numerical variables, depending upon normal distribution. Chi-square test or Fisher's Exact test was performed to assess categorical variables. The cutoffvalue of RDW (red cell distribution width), LDH (lactate dehydrogenase), albumin and CRP/albumin (C-reactive protein/albumin) in predicting mortality was analysed as Receiver Operating Characteristics (ROC). To explore the risk factors associated with mortality of COVID-19, univariable and multivariate logistic regression models were used. In the binary logistic regression analysis, mortality related parameters (diabetes mellitus, RDW, albumin, LDH, CRP/Albumin, AKI stage, age and gender) were included in the first step of the model, and the backward (Backward LR) selection method was used. A p-value of less than 0.05 was accepted asstatistically significant.
Ethics Committee approval from Sakarya University Medical Faculty was provided for this study (Approval No. 71522473/ 050.01.04/124).

\section{RESULTS}

During the aforementioned period, 131 patients were hospitalised with a preliminary diagnosis of COVID-19, and consulted the Nephrology Department for renal replacement therapy, were analysed. Seventy-six of these patients were excluded due to chronic renal failure. The remaining 55 patients, who followed a critical course, managed in the ICU and developed AKI, were included in the study. The mean age was 74 (64-80) years, and 36 $(65.5 \%)$ of patients were males (Table I). The most common symptoms at admission were shortness of breath (42 patients, $76.4 \%$ ) and cough (20 patients, 36.4\%). The most common comorbidities were hypertension (70.9\%) and diabetes mellitus (DM) (38.2\%). Clinical characteristics of the patients and laboratory results at admission are presented in detail in Tablel.

Thirty patients (54.5\%) had elevated creatinine values at admission, and 25 (45.5\%) developed AKI during ICU stay. According to the KDIGO clinical practice guidelines for acute kidney injury, 6 patients (10.9\%) developed stage 1, 13(23.6\%) developed stage 2 , and $36(65.5 \%)$ developed stage 3 AKI. Thirty-six $(65.5 \%)$ of the patients received renal replacement therapy: 16 (29.1\%) with intermittent hemodialysis, and 20 (36.4\%) with continuous renal replacement therapy (CRRT). During hospitalisation, 38 $(69.1 \%)$ out of 55 patients were intubated, 43 (78.2\%) died and $12(21.8 \%)$ patients were discharged with cure.

Comparison of survivors and nonsurvivors revealed that there was no statistically significant difference with respect to age and gender. However, survivors had a greater rate of DM as a comorbidity ( $p<0.05$, Table I). The comparison of two groups for laboratory studies showed a statistically significant difference in RDW, $\mathrm{LDH}$ and CRP/albumin on samples obtained at admission ( $p$ $<0.05$, Table I). In the nonsurvivor group, RDW and albumin levels at admission were lower, LDH and CRP/albumin values were higher. In the ROC analysis to determine the threshold levels, the threshold levels were defined as follows: RDW $17.70 \%$ (AUC: $0.718 ; 95 \% \mathrm{Cl}: 0.580-0.857 ; \mathrm{P}=0.022$ ) with $73.8 \%$ sensitivity and $66.7 \%$ specificity, albumin $34.30 \mathrm{gr} / \mathrm{L}$ (AUC: $0.741 ; 95 \%$ Cl: $0.570-0.912 ; P=0.014)$ with $83.3 \%$ sensitivity and $63.6 \%$ specificity, LDH 355.5 U/L (AUC: 0.731; 95\% Cl: $0.590-0.872 ; \mathrm{P}=$ 0.016 ) with $68.3 \%$ sensitivity and $66.7 \%$ specificity, CRP/albumin 1.37 (AUC: 0.721 ; \%95 Cl: $0.530-0.912 ; \mathrm{P}=0.025$ ) with $73.8 \%$ sensitivity and $72.7 \%$ specificity (Figure 1 ).

There was a statistically significant relationship between mortality and AKI stage $(p=0.030)$. Stage 3 patients had a higher mortality rate compared to stage 2 patients (32 patients, $88.9 \%$ vs. 7 patients, $53.8 \%$, respectively) ( $p=0.014)$. Length of ICU stay was not statistically different between the two groups; whereas, the length of stay in the inpatient unit was significantly shorter in the nonsurvivorgroup. The median length of stay in the inpatient unit was 3 ( 2 - 4) days in the nonsurvivor group and 6.5 (3.25 - 8.75) days in the survivorgroup $(p=0.005)$. 
Table I: Clinical characteristics and laboratory findings of the patients at admission.

\begin{tabular}{|c|c|c|c|c|}
\hline & All patients $(n=55)$ & Nonsurvivor $(n=43)$ & Survivor $(n=12)$ & p-value \\
\hline Age (years) ${ }^{*}$ & $74(64-80)$ & $75(65-81)$ & $65.5(55-79.25)$ & $0.162^{\mathrm{a}}$ \\
\hline Male sex $n(\%)$ & $36(65.5 \%)$ & $31(72.1 \%)$ & $5(41.7 \%)$ & $0.084^{b}$ \\
\hline \multicolumn{5}{|l|}{ Comorbıdıty n(\%) } \\
\hline Diabetes & $21(38.2 \%)$ & $13(30.2 \%)$ & $8(66.7 \%)$ & $0.041^{b}$ \\
\hline Hypertension & $39(70.9 \%)$ & $33(76.7 \%)$ & $6(50 \%)$ & $0.086^{b}$ \\
\hline Coronary artery disease & $19(34.5 \%)$ & $16(37.2 \%)$ & $3(25 \%)$ & $0.511^{b}$ \\
\hline NLR* & 7.47 (3.83-13.73) & $6.5(3.03-13.19)$ & $10.64(6.60-19.96)$ & $0.058^{\mathrm{a}}$ \\
\hline RDW, \%* & $16.25(15.0-19.05)$ & $16.0(14.87-18.25)$ & $18.15(16.12-20.57)$ & $0.022^{a}$ \\
\hline Basal cre, mg/dL* & $0.87(0.75-0.98)$ & $0.89(0.75-1.0)$ & $0.79(0.74-0.88)$ & $0.050^{\mathrm{a}}$ \\
\hline Cre at admission, mg/dL* & $1.15(0.86-1.78)$ & $1.06(0.86-1.59)$ & $1.49(0.92-2.14)$ & $0.267^{\mathrm{a}}$ \\
\hline Peak cre, mg/dL* & $3.27(2.07-4.5)$ & $3.28(2.76-4.48)$ & $2.07(1.61-4.51)$ & $0.117^{\mathrm{a}}$ \\
\hline Albumin, gr/L mean $\pm s d$ & $31.53 \pm 4.92$ & $30.60 \pm 4.41$ & $35.07 \pm 5.33$ & $0.006^{c}$ \\
\hline $\mathrm{LDH}, \mathrm{U} / \mathrm{L}^{*}$ & $383(288.5-504.0)$ & $405(317-555.5)$ & $324(225.5-379.3)$ & $0.016^{\mathrm{a}}$ \\
\hline $\mathrm{CRP}, \mathrm{mg} / \mathrm{L}^{*}$ & $74.2(27-166)$ & $83(32.96-169.44)$ & $31.9(9.37-109.01)$ & $0.050^{\mathrm{a}}$ \\
\hline CRP/alb* & $2.64(0.88-5.39)$ & $2.92(1.26-5.49)$ & $0.50(0.25-3.05)$ & $0.025^{\mathrm{a}}$ \\
\hline D-dimer, ng/mL* & $1640(715-4620)$ & $1640(715-4760)$ & $1540(579-3050)$ & $0.409^{\mathrm{a}}$ \\
\hline Lymphocyte, $x 10^{9} / L^{*}$ & $0.99(0.72-1.47)$ & $1.05(0.72-1.85)$ & $0.91(0.60-1.01)$ & $0.105^{\mathrm{a}}$ \\
\hline Procalcitonin, ng/ml & $0.5(0.18-1.31)$ & $0.45(0.18-1.32)$ & $0.78(0.26-1.7)$ & $0.732^{\mathrm{a}}$ \\
\hline \multicolumn{5}{|l|}{ Length of Hospital Stay* } \\
\hline Total days & $13(8-20)$ & $13(8-18)$ & $17(9.5-23)$ & $0.293^{\mathrm{a}}$ \\
\hline Inpatient unit days & $3(2-6)$ & $3(2-4)$ & $6.5(3.25-8.75)$ & $0.005^{\mathrm{a}}$ \\
\hline Intensive care days & $9(5-17)$ & $9(6-17)$ & $6.5(3.25-16)$ & $0.279^{\mathrm{a}}$ \\
\hline
\end{tabular}

Table II: Predictors of nonsurvivor COVID-19 patients using logistic regression models.

\begin{tabular}{|c|c|c|c|c|}
\hline \multirow{2}{*}{ Covariate } & \multicolumn{2}{|l|}{ Univariate } & \multicolumn{2}{|l|}{ Multivariate } \\
\hline & OR (95\% Cl) & $\mathbf{p}$ & OR (95\% Cl) & $\mathbf{p}$ \\
\hline Age & $1.044(0.988-1.103)$ & 0.128 & & \\
\hline Gender (Male) & 3.617 (0.959-13.635) & 0.058 & & \\
\hline DM (Positive) & $0.217(0.055-0.849)$ & 0.028 & & \\
\hline RDW (< 17.7\%) & $5.636(1.413-22.479)$ & 0.014 & & \\
\hline Albumin (<34.3 gr/L) & $8.750(2.007-38.144)$ & 0.004 & $12.793(1.847-88.614)$ & 0.010 \\
\hline LDH (> 355.5 U/L) & $4.308(1.096-16.930)$ & 0.036 & $8.454(1.299-55.023)$ & 0.026 \\
\hline CRP/alb (> 1.37) & 7.515 (1.686-33.499) & 0.008 & & \\
\hline AKI (stage 3) & $5.818(1.461-23.172)$ & 0.013 & $10.268(1.451-72.653)$ & 0.020 \\
\hline
\end{tabular}



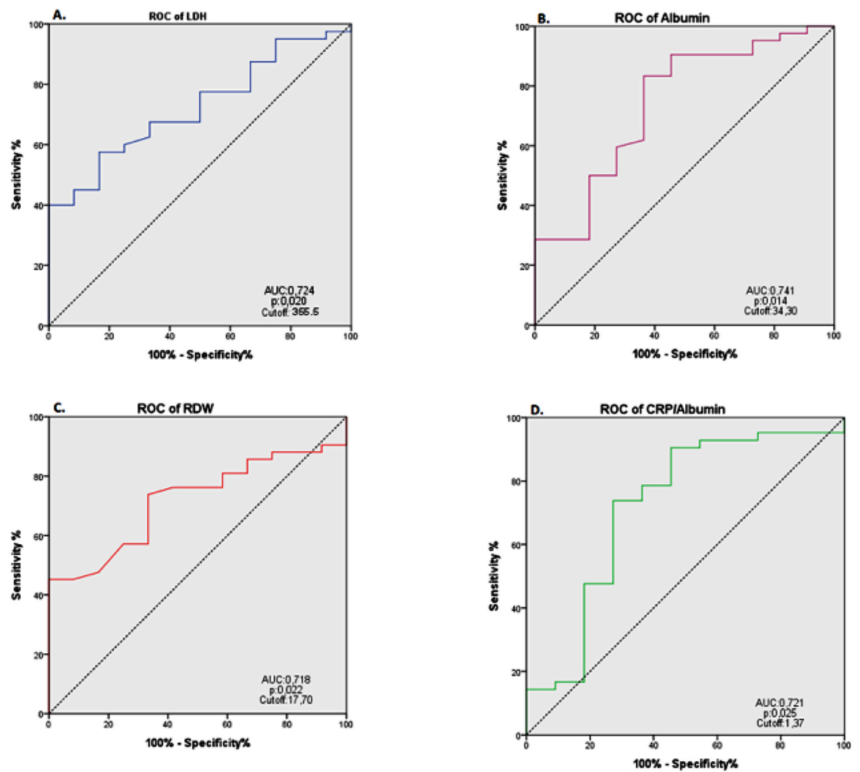

Figure 1: ROC analyses of RDW, LDH, and CRP/albumin values that may be predictors of mortality in patients with AKI.

(AUC: Area under the curve; LDH: Lactate dehydrogenase; RDW: Red cell distribution width; CRP/albumin: C-reactive protein/albumin).

The median length of ICU stay was 9 (6 - 17) days in the nonsurvivor group and $6.5(3.25-16)$ days in the survivor group $(p=0.279)$.

Age, sex, presence of DM, RDW (17.70\%), albumin $(<34.30$ $\mathrm{g} / \mathrm{dL}$ ), LDH (>355.5 U/L), CRP/albumin ratio (>1.37), and presence of stage $3 \mathrm{AKI}$ were assessed in the multivariability logistic regression model. As a result, low albumin level (OR: $12.79395 \% \mathrm{Cl}: 1.847-88.614, \mathrm{p}=0.010)$, high LDH level (OR: 8.454, 95\% Cl: $1.299-55.023, p=0.026)$, and presence of stage 3 AKI (OR: 10.268, 95\% Cl: $1.451-72.653, p=$ 0.020 ) were found as independent risk factors for mortality in COVID-19 patients who developed AKI (Table II).

\section{DISCUSSION}

AKI is a common complication in critically ill COVID-19 patients and an independent risk factor for mortality. If AKI and related deaths can be predicted, then the prognosis of patients may be improved by the application of specific treatments, providing adequate hemodynamic support, and avoiding nephrotoxic drugs. Therefore, detection of mortality predictors in patients with AKI is crucial.

AKI is associated with particularly high mortality of patients in the ICU. ${ }^{2}$ Cheng et al. reported a $16.1 \%$ mortality rate in COVID-19 patients, ${ }^{6}$ and a meta-analysis of 26 studies showed that patients with AKI had 13 -fold increased mortality. ${ }^{10}$ In this study, 43 (78.2\%) of the patients with AKI died. The high mortality rate was attributed to the inclusion of critically ill patients treated in the ICU. Similarly, a study that included 59 critically ill patients reported a mortality rate of $71.19 \%{ }^{11}$

Analysis of blood samples taken at admission showed that the nonsurvivor group in the present study had significantly lower RDW and albumin levels, higher LDH levels, and higher CRP/albumin ratios compared to the survivors. It was demonstrated for the first time that there could be a relationship between mortality and high CRP/albumin ratios or low RDW levels in COVID-19 patients, who developed AKI.

Decreased lymphocyte counts, elevated levels of CRP, sedimentation, ferritin, and D-dimer are among the basic laboratory findings of a COVID-19 infection. There was no significant difference in the lymphocyte counts, CRP, and D-dimer values at admission between the survivors and nonsurvivors in this study. On the other hand, a higher CRP/albumin ratio was found in nonsurvivors, a finding that was not encounterd in previous literature. The threshold level for CRP/albumin was determined as 1.37 , and there was no statistical significance in the regression analysis.

RDW levels were lower in nonsurvivors, and the RDW threshold level was $17.7 \%$. A study that compared community acquired pneumonia with COVID-19 cases found that RDW was one of the most significant distinguishing parameters, and COVID-19 patients had lower RDW levels compared with community acquired pneumonia patients. ${ }^{12}$ In that study, the threshold value for RDW was $13.35 \%$ (sensitivity $79.8 \%$ and specificity $84.6 \%$ ). In another study, Wang et al. found that patients with a more severe course had higher RDW-CV (red cell volume distribution width-coefficient of variation) and RDW-SD (red cell volume distribution width-standard deviation) compared to the group with moderate severity. ${ }^{13}$

In COVID-19 patients, the albumin levels measured at admission could be an independent risk factor and predict disease severity. ${ }^{14,15}$ A study, analysing AKI patients, found that low serum albumin level was an independent risk factor for stage 3 AKI, and levels of albumin at admission were lower in patients who developed stage 3 AKI. ${ }^{16}$ Similar to previous studies, it was found that low albumin level was an independent risk factor for COVID-19 patients, who developed AKI; and the threshold level for albumin was determined as $34.30 \mathrm{~g} / \mathrm{L}$.

LDH enzyme is found in all cells, especially in the myocardium and liver, its blood level is affected by various factors. Numerous studies have shown that it is an independent risk factor for severe disease and mortality. ${ }^{17,18}$ Similar to previous studies, LDH levels measured at admission were higher in nonsurvivors, and levels above $355.5 \mathrm{U} / \mathrm{L}$ constituted an independent risk factor for mortality in COVID-19 patients who developed AKI.

Studies also found that increased basal serum creatinine levels were associated with increased AKI. ${ }^{4,6,10}$ Moreover, patients with higher basal creatinine levels had higher rates of ICU admissions and mechanical ventilation requirements. ${ }^{6}$ Contrary to previous studies, Pelayo et al. found that the group which developed renal failure in the hospital had 
increased vasopressor and intubation needs, and increased mortality. ${ }^{19}$ Among the 55 patients included in this study, 30 (54.5\%) developed AKI at admission, and 25 (45.5\%) during ICU stay. There was no statistically significant difference between the groups.

In a study that assessed the relationship between $\mathrm{AKI}$ staging and mortality, patients at stage 3, according to the KDIGO guidelines, had increased mortality compared to stages 1 and $2{ }^{16}$ In this study, a statistically significant relationship was detected between stage and mortality. Especially stage 3 patients ( $88.9 \%$ mortality) had greater mortality compared to stage 2 patients (53.8\% mortality).

The presence of DM has been associated with a poorer prognosis and higher mortality in COVID-19 patients. ${ }^{20}$ Similar to others, in this study it was found that the most common comorbidity was hypertension (70.9\%), followed by diabetes $(38.2 \%)$. In contrast to other studies, a comparison of the comorbidities between the survivors and nonsurvivors revealed a higher rate of DM as a comorbidity in survivors.

During the clinical follow-up, there were no differences between the two groups with respect to the treatments applied for COVID-19 or length of stay in the ICU. However, the nonsurvivor group had a shorter length of stay in the inpatient unit. This was attributed to the early ICU need in nonsurvivor patients.

This study has some limitations. The inclusion of only patients managed in the ICU and the limited number of patients are the most significant of these limitations. Another limitation is the absence of RT-PCR positivity in all patients, the diagnosis was made with chest CT findings in some patients. Nevertheless, this is the first study that aims to determine the predictors of mortality in COVID-19 patients, who developed AKI.

\section{CONCLUSION}

Mortality is particularly high in COVID-19 infection associated AKI. Early diagnosis of the development of AKI and application of the necessary precautions may decrease morbidity, mortality, and possibly costs. It is found that low albumin and high LDH at admission and stage 3 AKI could be used as predictors of mortality in patients with COVID-19 pneumonia, who developed AKI. Moreover, it was demonstrated for the first time that a high CRP/albumin ratio and low RDW at admission could be associated with mortality in COVID-19 patients. More comprehensive studies are needed to confirm the reliability of this data.

\section{ACKNOWLEDGEMENT:}

We would like to thank all the medical, nursing, and technical staff members from the dialysis centres of Sakarya University Education and Research Hospital, for their dedicated care to the dialysis patients during the COVID-19 pandemic.

\section{ETHICAL APPROVAL:}

Approval of the Ethics Committee of Sakarya University Medical Faculty Ethics Committee approval code: 71522473/050.01.04/124, dated 20.03.2020 was obtained.

\section{PATIENTS' CONSENT}

This study was conducted in accordance with the Declaration of Helsinki. Written informed consents of all participants were obtained.

\section{CONFLICT OF INTEREST:}

The authors declared no conflict of interest.

\section{AUTHORS' CONTRIBUTION:}

AT: Agreement to be accountable for all aspects of the work in ensuring that questions related to the accuracy or integrity of any part of the work are appropriately investigated and resolved

HD, MB, SBA: Substantial contributions to the conception or design of the work; or the acquisition, analysis, or interpretation of data for the work.

OK: Drafting the work or revising it critically for important intellectual content.

SS: Final approval of the version to be published.

\section{REFERENCES}

1. Wang D, Hu B, Hu C, Zhu F, Liu X, Zhang J, et al. Clinical characteristics of 138 hospitalized patients with 2019 novel coronavirus-infected pneumonia in Wuhan, China. JAMA 2020; 323(11):1061. doi: 10.1001/jama.2020.1585.

2. Rabb H. Kidney diseases in the time of COVID-19: Major challenges to patient care. J Clin Invest 2020; 130(6):2749-51. doi: 10.1172/JCl138871.

3. Huang C, Wang Y, Li X, Ren L, Zhao J, Hu Y, et al. Clinical features of patients infected with 2019 novel coronavirus in Wuhan, China. Lancet 2020; 395(10223):497-506. doi: 10.1016/S01406736(20)30183-5.

4. Hirsch JS, Ng JH, Ross DW, Sharma P, Shah HH, Barnett RL, et al. Acute kidney injury in patients hospitalised with COVID-19. Kidney Int 2020; 98(1):209-18. doi: 10.1016/j.kint.2020.05.006.

5. Zhou P, Yang XL, Wang XG, Hu B, Zhang L, Zhang W, et al. A pneumonia outbreak associated with a new coronavirus of probable bat origin. Nature 2020; 579(7798):270-3. doi: 10.1038/s41586020-2012-7.

6. Cheng Y, Luo R, Wang K, Zhang M, Wang Z, Dong L, et al. Kidney disease is associated with in-hospital death of patients with COVID-19. Kidney Int 2020; 97(5):829-38.

7. Ronco C, Reis T. Kidney involvement in COVID-19 and rationale for extracorporeal therapies. Nat Rev Nephrol 2020; 16(6):308-10. doi: 10.1038/s41581-020-0284-7.

8. Qu JM, Wang C, Cao B. Guidance for the management of adult patients with coronavirus disease 2019. Chin Med J (Engl) 2020; 133(13):1575-94. doi: 10.1097/CM9.000000000000089

9. Kellum JA, Lameire N, Aspelin P, Barsoum RS, Burdmann EA, Goldstein $\mathrm{SL}$, et al. Kidney disease: Improving global outcomes (KDIGO) acute kidney injury work group. KDIGO clinical practice guideline for acute kidney injury. Kidney Int Suppl 2012; 2(1):1-138. doi: 10.1186/cc11454.

10. Hansrivijit P, Qian C, Boonpheng B, Thongprayoon C, Vallabhajosyula $\mathrm{S}$, Cheungpasitporn W, et al. Incidence of acute kidney 
injury and its association with mortality in patients with COVID-19: A meta-analysis. J Investig Med 2020; 68(7):1261-70. doi: 10.1136/jim-2020-001407.

11. Guan W, Ni Z, Hu Y, Liang W, Ou C, He J, et al. Clinical characteristics of coronavirus disease 2019 in China. N Engl J Med 2020; 382(18):1708-20. DOI: 10.1056/NEJMoa2002032.

12. Pan $Y, Y$, $G$, Zeng $X$, Liu $G$, Zeng $X$, Jiang $X$, et al. Can routine laboratory tests discriminate SARS-CoV-2-infected pneumonia from other causes of community-acquired pneumonia? Clin Transl Med 2020; 10(1):161-8. doi: 10.1002/ctm2.23.

13. Wang C, Deng R, Gou L, Fu Z, Zhang X, Shao F, et al. Preliminary study to identify severe from moderate cases of COVID-19 using combined hematology parameters. Ann Transl Med 2020; 8(9):593-593. doi: 10.21037/atm-20-3391.

14. Aziz M, Fatima R, Lee-Smith W, Assaly R. The association of low serum albumin level with severe COVID-19: A systematic review and meta-analysis. Crit Care 2020; 24(1):255. doi: 10.1186/s13054- 020-02995-3.

15. Violi F, Cangemi R, Romiti GF, Ceccarelli G, Oliva A, Alessandri F, et al. Is Albumin Predictor of Mortality in COVID-19? Antioxid Redox Signal 2020; ars 2020.8142. doi: 10.1089/ars.2020.8142.
16. Lim JH, Park SH, Jeon $\mathrm{Y}, \mathrm{Cho} \mathrm{JH}$, Jung HY, Choi JY, et al. Fatal outcomes of COVID-19 in patients with severe acute kidney injury. J Clin Med 2020; 9(6):1718. doi: 10.3390/jcm9061718.

17. Han $\mathrm{Y}$, Zhang H, Mu S, Wei W, Jin C, Tong C, et al. Lactate dehydrogenase, an independent risk factor of severe COVID-19 patients: A retrospective and observational study. Aging (Albany NY) 2020; 12(12):11245-58. doi: 10.18632/aging.103372.

18. Bousquet G, Falgarone G, Deutsch D, Derolez S, Lopez-Sublet M, Goudot F-X, et al. ADL-dependency, D-Dimers, LDH and absence of anticoagulation are independently associated with one-month mortality in older inpatients with Covid-19. Aging (Albany NY) 2020; 12(12):11306-13. doi: 10.18632/aging.103583.

19. Pelayo J, Lo KB, Bhargav R, Gul F, Peterson E, Dejoy III R, et al. Clinical characteristics and outcomes of community- and hospital-acquired acute kidney injury with COVID-19 in a US inner city hospital system. Cardiorenal Med 2020; 10(4):223-31. doi: 10.1159/000509182.

20. Huang I, Lim MA, Pranata R. Diabetes mellitus is associated with increased mortality and severity of disease in COVID-19 pneumonia - A systematic review, meta-analysis, and meta-regression. Diabetes Metab Syndr Clin Res Rev 2020; 14(4):395-403. doi: 10.1016/j.dsx.2020.04.018. 